Meta

Journal des traducteurs

Translators' Journal

\title{
Le péché de « nivellement » dans la traduction littéraire
}

\section{Françoise Wuilmart}

Volume 52, numéro 3, septembre 2007

URI : https://id.erudit.org/iderudit/016726ar

DOI : https://doi.org/10.7202/016726ar

Aller au sommaire du numéro

\section{Éditeur(s)}

Les Presses de l'Université de Montréal

\section{ISSN}

0026-0452 (imprimé)

1492-1421 (numérique)

Découvrir la revue

Citer cet article

Wuilmart, F. (2007). Le péché de " nivellement » dans la traduction littéraire. Meta, 52(3), 391-400. https://doi.org/10.7202/016726ar

\section{Résumé de l'article}

Un texte d'auteur s'écarte par définition de la norme langagière et acquiert un certain « relief littéraire ».

Un des dangers qui guette le traducteur non averti est le nivellement de ce relief complexe. Un autre danger est la recréation réductrice, c'est-à-dire réduite à une prise de sens individuelle et à une réécriture soucieuse d'adaptation et donc mutilante.

Le texte d'auteur est polysémique et son nivellement peut se produire à plusieurs niveaux : lexical et stylistique, culturel, et idéel.

Le traducteur littéraire de bon aloi devrait donc se libérer du carcan de la langue d'arrivée et la traiter comme un organe vivant, susceptible d'accueillir l'Autre de l'auteur, et l'Autre de la culture source. 


\title{
Le péché de «nivellement » dans la traduction littéraire
}

\author{
FRANÇOISE WUILMART \\ Haute École de Bruxelles, Bruxelles, Belgique \\ ctls@skynet.be
}

\begin{abstract}
RÉSUMÉ
Un texte d'auteur s'écarte par définition de la norme langagière et acquiert un certain «relief littéraire».

Un des dangers qui guette le traducteur non averti est le nivellement de ce relief complexe. Un autre danger est la recréation réductrice, c'est-à-dire réduite à une prise de sens individuelle et à une réécriture soucieuse d'adaptation et donc mutilante.

Le texte d'auteur est polysémique et son nivellement peut se produire à plusieurs niveaux: lexical et stylistique, culturel, et idéel.

Le traducteur littéraire de bon aloi devrait donc se libérer du carcan de la langue d'arrivée et la traiter comme un organe vivant, susceptible d'accueillir l'Autre de l'auteur, et l'Autre de la culture source.
\end{abstract}

\begin{abstract}
An author's text, by definition, deviates from ordinary language and acquires a certain "literary shape." One of the dangers lying in wait for the inexperienced translation is the standardization of this complex shape. Another is simplistic re-creation, reduced to capturing individual meaning and to a careful rewriting which is adaptive and thus disfiguring.

An original text is polysemous, and levelling can occur at several levels: lexical, stylistic, cultural and conceptual. A good literary translator must break free from the grip of the target language, using it as a living instrument which can accommodate the author's distinctness and that of the source culture.

\section{MOTS-CLÉS/KEYWORDS}

relief littéraire, polysémie, nivellement, ciblisme excessif, langue modulable
\end{abstract}

Le phénomène de nivellement touche au cœur même du problème de toute traduction littéraire. Nivellement, ou encore «normalisation», c'est-à-dire action de «raboter» un texte ou de l'aplatir: y supprimer toutes les sortes de reliefs, y tronquer les pointes, y boucher les creux, y aplanir toutes les aspérités qui en font justement un texte littéraire.

Une telle approche de la traduction littéraire, poétique surtout, est encore bien trop courante. Le «traducteur-niveleur» ne peut être un grand écrivain, car ce qu'il craint précisément c'est de prendre trop de libertés, alors que l'auteur ne construit sa parole qu'en s'écartant de la norme: en retournant à l'étymologie, en ravivant des racines enfouies, en forgeant des mots nouveaux, en bouleversant la syntaxe, en jouant sur les connotations multiples, et aussi en donnant à son texte une dimension polysémique, marque de fabrique, label de qualité de toute grande écriture. C’est parce qu'un texte est polysémique qu'il survit à son époque et que les générations suivantes y découvrent des éléments qui les concernent toujours, et qui avaient échappé aux contemporains de l'auteur. Songeons à Shakespeare, «notre éternel contemporain», 
comme il fut maintes fois baptisé. Le traducteur-niveleur, lui, n'a qu'une lecture réductrice du texte d'auteur, car il le lit dans l'optique restreinte non seulement d'une époque, mais d'un milieu, le sien. Pire encore: la lecture qu'il en fait passe par le prisme étroit de son propre vécu, et il ne retiendra du texte que ce qui le touche ou le concerne personnellement. Il est vrai que tout texte cesse d'appartenir à son auteur dès lors qu'il a quitté sa table de travail. Une fois publié et livré en pâture à la foule des lecteurs, il devient protéiforme. Le texte littéraire est par essence objet d'interactivité: la page est lue, assimilée, décodée par le récepteur, et ce mariage intime du «dit-imprimé» et du «lu-ressenti», cette véritable combinaison chimique peut être très féconde: car ce qui en naît, c'est un texte autre, qui ne reflète pas nécessairement l'image que s'en faisait l'auteur en le rédigeant. Tout lecteur peut d'ailleurs lire un même texte de manières différentes selon l'âge, et même selon l'heure du jour. C'est une expérience inévitable que nous connaissons tous. Là où cela peut avoir des conséquences fâcheuses, c'est dès qu'il s'agit non plus de le lire, mais de le "recréer", c'est-à-dire de transposer ce qui est lu dans une autre langue, une autre culture. À ce stade-ci, le traducteur idéal devrait pouvoir restituer toute la polyvalence du texte original. Tâche bien ardue, il est vrai, que notre traducteur-niveleur préfère ignorer, car la "fidélité» telle qu'il la conçoit est tout autre: fidèle il l'est d'abord à lui-même, lisant le texte avec le regard du «je» et non d'un «il», celui de l'auteur, ou d'un «nous» présent et à venir, celui des contemporains et des générations futures. Mais fidèle il l'est aussi à une certaine conception de sa propre langue. Pour lui, un texte littéraire est d'abord un texte «bien écrit», «bien léché», par quoi il entend: écrit dans une langue plutôt classique, traditionnelle, correcte, et généralement émaillées de clichés stylistiques, tristes vestiges d'énoncés jadis originaux. Je me rappelle le cas évocateur de cet étudiant qui se prétendait excellent traducteur du seul fait que le français de ses traductions était toujours impeccable. Ce qu'il refusait de reconnaître c'est que, quel que soit l'auteur italien qu'il transposait, le style français demeurait invariablement le même. Il «n'entendait pas» la voix du texte, car la voix qui guidait sa plume était toujours la sienne propre, ou celle d'un éventuel professeur de français rigoureux qu'il n’avait jamais réussi à évacuer de son esprit.

La conception que le traducteur-niveleur a de sa langue est donc étroite, elle n'est en rien créatrice, et il ne devrait jamais s'attaquer à de grands auteurs, incapable qu'il est de bouleverser des schémas figés. Cependant, l'effet normalisateur dont il est responsable est sans doute encore involontaire, inconscient, car à côté de lui, il est une autre espèce de traducteur-niveleur parfaitement conscient et de plus grande envergure. Au risque de choquer certains de ses admirateurs, je prendrai comme exemple un Alexandre Vialatte, avec sa traduction de Kafka. Alors que le texte français passe pour être remarquable et que l'on doit sans doute à ce grand traducteur le mérite de nous avoir fait découvrir Kafka, la version française est bien loin du texte allemand, aucun germaniste ne le contestera. On peut dire que Vialatte produit justement ce français «léché» fait de phrases bien balancées, souvent plus longues que celles de l'original, et où le traducteur privilégie l'élégance et le rythme français, apanage de cette langue «de cour». Nous y sommes donc très loin du véritable Kafka dont la langue est pure et dure, simple mais rigoureuse et précise, parfois cinglante, et justement ambiguë, si bien qu'on le lit aujourd'hui autrement et que l'on veut y voir une dimension d'humour qui n'est pas assez développée chez Vialatte. Son Kafka est francisé à l'extrême, car l'intention du traducteur est ici d'être fidèle à la langue d'arrivée. 
Vialatte était ce que Jean-René Ladmiral appellerait un pur «cibliste». La francisation du texte allemand, son adaptation à la culture d'arrivée est une forme de nivellement que j'appellerais volontiers "intolérant», voire nationaliste. Aujourd'hui, on a heureusement compris qu'il ne fallait plus traduire ainsi. Ce qui n'empêche que le texte de Vialatte a un charme certain, car il est des traducteurs-niveleurs qui, comme lui, tiennent certes du génie.

Pour en revenir aux diverses facettes de l'action nivelante, il me semble qu'il faut la diviser en trois catégories:

\section{Le nivellement culturel}

Le plus grave sans doute, et le plus intolérant. On l'oublie trop souvent: traduire un texte, c'est aussi traduire une culture. La langue de départ reflète une vision du monde qui peut être aux antipodes de celle de la culture d'arrivée: ainsi, et comme se plaisait à le souligner Nietzsche, l'allemand est-il la langue du devenir, tandis que le français serait celle de l'être. Cette différence fondamentale se décante à tous les niveaux linguistiques ou stylistiques: dans la conjugaison, la syntaxe, les connotations, le rythme même des phrases. L'approche du temps, plus particulièrement, se reflète avec précision dans les temps ou les modes de la conjugaison qui peut être très complexe, comme en anglais, ou simplissime comme en allemand. Comment dès lors transmettre et faire passer les nuances temporelles dans une langue qui ne dispose pas des outils nécessaires pour les accueillir et les reproduire? Cette différence peut culminer dans les grandes métaphores symboliques: le soleil qui est du masculin en français est du féminin en allemand, et nous savons que tout un poème peut être basé sur le sexe de l'astre et ses attributs, cas extrême où la traduction devient carrément impossible, si ce n'est au prix de contorsions infinies.

Dans ce cadre-ci, un cas typique de nivellement qui est source de nombreux débats, est celui du rendu d'éléments culturels dits « exotiques». Prenons le poème où Prévert évoque le cerisier, et imaginons sa traduction en hindi. Faut-il remplacer le cerisier par un arbre local sous prétexte d'éveiller chez le lecteur indien des sentiments familiers et analogues? Ou faut-il garder le cerisier français et amener ainsi le lecteur étranger à le découvrir? Pour ma part, j'opterais plutôt pour la seconde solution, précisément pour sa dimension d'ouverture à ce qui est étranger. Et puis si Prévert a choisi le cerisier, toutes les vertus poétiques et symboliques qu'il place dans cet arbre sont absolument intransposables dans une autre plante. Adapter les ingrédients culturels à la culture d'arrivée, c'est faire fi de l'âme même de la culture étrangère, c'est une action réductrice et une forme de nivellement que l'on pourrait qualifier d'éthiquement blâmable.

\section{Le nivellement stylistique}

Revenons au fameux relief du texte littéraire, a fortiori poétique. C'est lui qui singularise la parole par rapport à la langue normative ou courante. L'auteur prend à l'égard de sa langue des libertés énormes, allant même jusqu'à en violer les règles les plus élémentaires ou à la défigurer, pour des raisons esthétiques ou idéologiques. Un des problèmes majeurs de la transposition littéraire est que le traducteur n'est pas toujours sensible à cet écart. L'auteur peut par exemple sélectionner parmi une série de 
synonymes le mot le moins courant, le moins «émoussé», choix qui a pour effet de conférer plus de force au concept ou à l'objet. Le traducteur qui aura une connaissance insuffisante de la langue de départ ne sera pas sensible à la rareté du mot à traduire, et le rendra par un terme courant dans la langue d'arrivée. Ce qui peut être grave si le terme choisi par l'auteur l'était par exemple pour ses connotations idéologiques ou historiques ouvrant dans le texte une dimension essentielle, commandant une certaine interprétation qui échappe au traducteur et donc au lecteur de la traduction. À un lexique "émoussé» dans la langue de départ, doit correspondre un vocabulaire également émoussé dans la langue d'arrivée. Inversement, un terme rare et fort, ne peut être traduit que par un terme rare et fort. C'est là un dosage trop souvent ignoré et qui est pourtant d'importance capitale.

Dans le même ordre d'idée, le traducteur-niveleur peut ne pas avoir capté le «ton juste» du texte original. Un peu comme un pianiste qui ignorerait le ton à la clé du morceau qu'il interprète, oubliant ici un bémol, là un dièse. Or, le «ton » du texte est responsable de sa cohérence. Qui dit texte, dit texture. Le traducteur qui ne rendrait pas ce ton à la clé risquerait de créer un non-texte dans la langue d'arrivée, avec par exemple des contradictions flagrantes entre le «dit», le signifié, et le «ton» du texte traduit qui serait fait d'une succession de fausses notes.

Cela nous amène à un troisième aspect du nivellement stylistique, le plus important sans doute: l'ignorance de la relation entre le contenu et la forme. En 1982, dans Un art en crise, essai poétique de la traduction poétique, Efim Etkind a bien résumé le problème. Voici ce qu'il y dit: «Le terme de traduction n'est vraiment pas précis. Traduire veut dire rendre le contenu de l'énoncé en une autre langue. Or, cette définition pose un autre problème, celui du terme "le contenu"; est-il aussi clair qu'il semble l'être? Pour des textes scientifiques, aussi bien du domaine de la philosophie que de ceux de la biologie et même de l'histoire, c'est évident: le contenu n'est que la somme d'informations rationnelles. La forme de l'expression joue un certain rôle extérieur, mais on peut l'ignorer. Nous avons donc toute une gamme de textes à partir de celui où le rôle que joue la forme verbale pour le contenu général est quasi nul, jusqu'à celui où c'est la forme qui elle-même devient le contenu». Nous y voilà: la forme devient elle-même le contenu. On pourrait recourir à une comparaison iconique: le texte non littéraire c'est la photographie pure et simple de la montagne SainteVictoire, le texte littéraire c'est la même montagne peinte par Cézanne, pleine de cônes, de sphères et de cubes. Décrire le tableau de Cézanne à un aveugle, en termes d'arbres, de pierres et de verdure, serait une trahison. Décrire au même aveugle la photo du paysage en termes de cylindres, de cubes et de sphères, en serait une autre, tout aussi grave. Nous voilà au cœur même du problème de la restitution fidèle, de la traduction de bon aloi. L'œuvre d'art n'existe que par la forme. Pour citer encore Efim Etkind lorsqu'il analyse la Chanson de Verlaine:

Les sanglots longs

Des violons

De l'automne

Blessent mon cœur

D'une langueur

Monotone ... 
Ce poème n'existe que par sa forme sonore. "Les sanglots» et «les violons de l'automne» ne possèdent qu'une valeur associative; toute tentative de s'imaginer à travers ces images des phénomènes du monde matériel est vouée à l'échec; ce n'est ni le vent, ni la pluie, ni la chute des feuilles, c'est plutôt un état d'âme inexprimable autrement que par ces symboles et à travers ces enchaînements de sonorités: «longsviolons, cour-langueur». Et Etkind de poursuivre: «Il est évident qu'en traduisant en une autre langue la Chanson de Verlaine, l'équivalent de ses associations et de ses enchaînements de sonorités est absolument nécessaire. Par contre la reproduction du sens des mots n'a presque aucune importance. Ce qui compte c'est la mélodie du poème et la tristesse comme atmosphère, et une autre forme d'exactitude serait une trahison. Toute traduction poétique est d'ailleurs une métatraduction: en recréant le poème on est obligé de puiser à des sources qui sont extratextuelles.»

Les traducteurs allemands de la Chanson de Verlaine l'ont très bien compris, puisqu'ils ont abandonné les violons et les sanglots pour privilégier la sonorité et ont choisi dans la langue allemande des sons qui évoquaient la même tristesse, la même mélancolie que l'original, sans se soucier des objets eux-mêmes.

Ce que l'auteur d'un texte littéraire veut « dire», est donc placé dans, véhiculé par bien autre chose que le signifié. Le «dit» du texte littéraire, et plus encore poétique, est une dynamique insaisissable; le texte est un tissu dont chaque maille retire sa valeur de son entrelacement avec d'autres mailles, où chaque couleur retire sa saturation des couleurs qui l'entourent. Et il faut sans doute y voir la raison majeure pour laquelle tout artiste, de quelque discipline que ce soit, est toujours irrité par la sempiternelle question du profane: «Qu'est-ce que vous avez voulu dire dans ce film? Ou dans ce poème?». Question bien peu pertinente puisque ce que l'artiste a voulu dire, il ne pouvait le dire que de la manière dont il l'a dit dans son œuvre précisément, et qu'il ne peut répondre que par et dans cette forme. Le «dit» du texte littéraire est unique, absolument unique.

Pour établir maintenant un parallèle révélateur: le langage corporel contredit parfois le sens des mots prononcés; un certain regard fuyant, un geste incontrôlé de la main peuvent infirmer de manière flagrante l'énoncé d'une phrase. La robe (le regard ou le geste) qui enveloppe la parole, ou encore le «geste de la voix», c'est-à-dire l'intonation, contaminent le signifié pur et l'extirpent du dictionnaire pour le faire participer à un ballet sémantique des plus complexes. C'est sur la même dialectique que se fonde le texte littéraire ou poétique. La structure mélodique, rythmique, la distribution subtile des sémantèmes ou des phonèmes, les connotations sonores entraînent les mots dans une vaste symphonie où toute valeur verbale est relativisée par rapport à l'ensemble. Le mot ici est mis au service de cet ensemble. Il est pesé, sélectionné pour des raisons diverses: entre autres pour sa longueur ou sa brièveté, pour ses références intertextuelles, ou enfin sa valeur sonore, comme le célèbre Nevermore d'Edgar Poe; mais dans tous les cas, il est irremplaçable et le soustraire à l'ensemble harmonisé équivaudrait à retrancher une pierre d'une mosaïque et aurait le même effet désastreux.

La valeur «esthétique» du signifiant est d'ailleurs si importante qu'elle peut même parfois infirmer le signifié, au point d'en arriver à cette situation paradoxale que la forme contredise le contenu! Ainsi dans le poème de Heinrich Heine, Meeresstille (littéralement: Calme en mer), où tous les expédients rythmiques et sonores, pour la plupart abrupts, hachés et rudes, évoquent exactement le contraire de l'harmonie marine. 
Voici d'abord le texte allemand:

\section{MEERESSTILLE}

Meeresstille! Ihre Strahlen

Wirft die Sonne auf das Wasser,

Und im wogenden Geschmeide

Zieht das Schiff die grünen Furchen.

Bei dem Steuer liegt der Bootsmann

Auf dem Bauch und schnarchet leise.

Bei dem Mastbaum, segelflickend,

Kauert der beteerte Schiffsjung.

Hinterm Schmutze seiner Wangen

Sprüht es rot, wehmütig zuckt es

Um das breite Maul, und schmerzlich

Schaun die grossen schönen Augen.

Denn der Kapitän steht vor ihm,

Tobt und flucht und schillt ihn Spitzbub'.

«Spitzbub'! einen Hering hast du

Aus der Tonne mir gestohlen!»

Meeresstille! Aus den Wellen

Taucht hervor ein kluges Fischlein,

Wärmt das Köpfchen in der Sonne,

Plätschert lustig mit dem Schwänzschen.

Doch die Möwe, aus den Lüften,

Schiesst herunter auf das Fischlein,

Unde den raschen Raub im Schnabel,

Schwingt sie sich hinauf ins Blaue.

Gérard de Nerval, ami et traducteur attitré de Heine, a fourni une traduction du poème curieusement approuvée par l'auteur lui-même:

La mer est calme. Le soleil reflète ses rayons dans l'eau, et sur la surface onduleuse et argentée le navire trace des sillons d'émeraude.

Le pilote est couché sur le ventre, près du gouvernail, et ronfle légèrement. Près du grand mât, raccommodant des voiles, est accroupi le mousse goudronné.

Sa rougeur perce à travers la crasse de ses joues, sa large bouche est agitée de tressaillements nerveux, et il regarde ça et là tristement avec ses grands beaux yeux.

Car le capitaine se tient devant lui, tempête et jure et le traite de voleur:

«Coquin! tu m'as volé un hareng dans le tonneau!»

La mer est calme. Un petit poisson monte à la surface de l'onde, chauffe sa petite tête au soleil et remue joyeusement l'eau avec sa petite queue.

Cependant, du haut des airs, la mouette fond sur le petit poisson, et, sa proie frétillante dans son bec, s'élève et plane dans l'azur du ciel.

Si j'ai tenu à citer d'abord cette traduction, c'est parce qu'elle éclaire parfaitement sur le contenu du poème, la scène et les deux événements qui se déroulent parallèlement: le capitaine qui se fâche contre le moussaillon parce que celui-ci lui a volé un hareng, et la mouette qui vient pêcher un petit poisson pour s'en retourner le déguster au ciel. Voilà un contenu bien peu poétique et un sujet bien pauvre. Toute la dimension 
poétique est dans la forme. Ce que l'on pourrait reprocher à la traduction de Nerval, c'est d'avoir mis à plat un contenu et d'avoir nivelé la forme à outrance, au point de devenir inexistante en français. Même la configuration du sonnet a disparu au profit de phrases prosaïques.

Le titre du poème et le premier mot annoncent un paysage marin, empreint de calme. Or, les deux événements cités n'ont rien de pacifique, au contraire, bien qu'ils relèvent du quotidien: un capitaine qui gronde un subalterne, un oiseau qui avale un poisson. Voilà une première contradiction, deux sortes de petites guerres au beau milieu de la grande bleue. On pourrait y voir aussi des allusions idéologiques: l'exploitation de la classe inférieure ou des enfants, par les chefs ou les adultes peu compréhensifs, voire inhumains; on pourrait établir des comparaisons d'ordre plus éthique: dans la nature il est permis de voler impunément un poisson pour se nourrir, mais pas dans la société où les rapports sont plus injustes, etc. Pourtant, tout cela resterait banal sans le message qui se situe véritablement au niveau de la forme: tout d'abord le rythme est constitué d'octosyllabes bien martelées; les assonances et les allitérations recourant principalement à des sifflantes, à des chuintantes, à des explosives; énormément de consonnes dures et sourdes qui s'entrechoquent brutalement. Nous avons affaire ici à un exemple parfait de forme infirmant le contenu: un contenu anodin, voire banal, exprimé dans une musique de rudesse et de révolte.

La traduction de Gérard de Nerval est une illustration parfaite du phénomène de nivellement, de rabotage, son texte français se réduit au contenu d'une histoire anodine qui mérite encore bien peu l'appellation de poème: tout le relief sonore de l'original en est absent. Je lui préfere de loin la traduction plus récente de Nicole Taubes qui a le mérite d'avoir restitué une sonorité et un rythme souvent proches de l'original:

\section{GRAND CALME EN MER}

Grand calme en mer! Les rayons

Du soleil se jettent sur les eaux,

Et dans l'ondoiement du joyau

Le bateau trace son sillon.

Le timonier, affalé, dort

En ronflant près du gouvernail;

Près du mât, ravaudant les voiles,

Maculé de goudron le mousse.

Sous ses joues salies, la rougeur,

Perce, un triste tremblement tord

Sa bouche animale, et ses yeux

Grands et beaux sont pleins de douleur.

Le capitaine est devant lui,

Qui crie furieux et l'injurie:

«Coquin! Scélérat! Tu m’as pris

Un hareng volé dans la tonne!»

Grand calme en mer! Mais hors des flots

Sort un gentil petit poisson,

Il chauffe au soleil son museau,

Gaiement de sa queue frappe l'eau. 
Mais la mouette fendant les airs, Fond comme un trait sur le poisson Et, sa proie dans le leste bec, S'élance et se perd dans le bleu

(Poème XI du Premier cycle de La Mer du Nord. Livre des Chants, p. 180. Éd. Le Cerf, collection Bibliothèque franco-allemande, 1999)

Un autre mérite de cette traduction est d'avoir respecté le champ sémantique géométrique du tableau en mer: si l'on représente les verbes de position et de mouvement par des lignes, on obtient des verticales, des horizontales et des obliques qui s'entrecoupent et strient le paysage constitué par la surface de la mer, l'horizon et le ciel (les rayons du soleil se jettent sur les eaux, le bateau trace son sillon, un personnage est affalé, l'autre est debout, la mouette fond comme un trait, s'élance dans le bleu, etc.). Comme on le constatera aussi, toutes ces lignes sont en mouvement, si bien que l'impression générale est celle d'éclairs qui s'entrecroisent et se précipitent chacun dans une direction différente, ce qui ne fait que renforcer le sentiment de mésentente, et évoque donc tout le contraire de l'harmonie annoncée dans le titre: car il n'y a aucune convergence entre ces lignes. Ce remarquable champ sémantique visuel correspond donc bien au paysage sonore du poème, lui aussi agressif. Toutes ces choses semblent avoir échappé à Gérard de Nerval, qui succombe bel et bien ici au «péché de nivellement»; sa traduction est une trahison, et l'on ne peut que s'étonner du fait que l'auteur l'ait approuvée.

\section{Le nivellement des idées}

Il est une troisième forme de nivellement qui serait plutôt d'ordre idéologique: ce qui échappe à bon nombre de traducteurs, c'est que le bouleversement, parfois révolutionnaire, exprimé par un texte, ne se traduit pas seulement dans le signifié, mais déjà au niveau du signifiant.

C'est ce que je voudrais démontrer maintenant à l'exemple d'un auteur, un philosophe poète que je connais bien pour l'avoir traduit pendant plus de 20 ans: Ernst Bloch. Dans son Principe Espérance, il y a adéquation parfaite entre la pensée et l'écriture. En quelques mots: les catégories philosophiques nouvelles et le système ouvert de Bloch ne pouvaient être exprimés que dans une langue elle aussi ouverte, novatrice, voire dérangeante, en tout cas suggestive et non discursive. L'écriture blochienne crevasse ou fait sauter le vernis des concepts clos, traditionnels, émoussés, elle bouleverse la syntaxe dite normale et applique à la structure du texte les principes de la composition musicale, car pour Bloch la musique est au faite de tous les arts. Les ingrédients de sa langue sont les mêmes que ceux de l'action éthique qu'il prône: la tension, l'énergie, la couleur, la subversion, le souffle prophétique qui bousculent et embrasent, et poussent à ce qu'il appelle «l'optimisme militant». De quelle manière? Tout d'abord, Bloch saisit pour ainsi dire la langue allemande à la racine. Il redonne vie à des éléments que le temps et l'usage courant avaient émoussés, faisant ressortir le sens originel qu'on avait oublié. Ce rappel fréquent de l'étymologie colore son texte et y réintroduit l'archétype et le mythe, le symbole ou l'allégorie. C'est ainsi qu'il substantive des adverbes, des conjonctions, des particules décolorées par l'usage quotidien, délavées par le temps: il réinvestit ces petits mots simples et purs de leur sens 
premier et fort. Par ailleurs, Bloch a une prédilection très marquée pour un type de structure qui peut se comparer au phénomène de l'écho: la structure binaire (FurchtEhrfurcht/ bewusst/gewusst), et il n'est pas nécessaire de connaître l'allemand pour apprécier l'impact rythmique de ces couples lexicaux. Toutefois, si l'effet d'écho est formellement et rythmiquement indéniable, le message énoncé dans la résonance s'écarte presque toujours de la première voix, il va plus loin ou s'y oppose carrément: parallèlement la philosophie blochienne est l'ennemie héréditaire de la stérile répétition, elle privilégie le dépassement, et le premier foyer de ce dépassement est l'écriture. Autre exemple: la jonglerie lexicale avec laquelle il compose ses variations presque musicales sur un thème de base, généralement un sémantème du type de wirklich (réel). Les modulations de ce mot réalisées par l'adjonction d'affixes divers ou par les flexions verbales (Verwirklichtsein - des Verwirklichenden - das Verwirklichte - die Aporien der Verwirklichung, etc.) correspondent toujours à une sorte de déclinaison de la réalité; dans le cas cité: aux diverses étapes du processus de la réalisation dans le matérialisme dialectique.

Enfin, dans le Principe Espérance, elles sont légions ces compositions verbales inattendues, ces assemblages déconcertants où l'auteur marie l'abstrait et le concret, comme lorsqu'il qualifie l'histoire de Don Quichotte de Prügelgeschichte des abstrakt Unbedingten: «histoire de bastonnade de l'Absolu abstrait». Le rapprochement constant d'éléments lexicaux disparates est toujours sous-tendu par un courant profond: celui de la force explosive, subversive, prométhéenne, jobienne ou mosaïque qui s'exprime très précisément dans cette langue inhabituelle faite d'expressions aberrantes et troublantes: c'est la langue de la Sortie ou de l'Exode. Les stéréotypes, les tournures familières sont défigurés pour que se fissure la surface des rapports traditionnels et conventionnels; de nouvelles combinaisons linguistiques créent des montages, parfois surréalistes, qui font naître de ces ensembles insolites un Nouveau possible, et l'éclosion du Nouveau réel est en effet une des catégories centrales de la philosophie blochienne.

En résumé: rythme, structure mélodique, scansion, métaphore, éclatement de concepts émoussés, bouleversement de la syntaxe traditionnelle, telles sont les composantes majeures de cette écriture qui mérite sans doute bien le qualificatif 'poétique'. C'est l'écriture elle-même qui fait déjà éclore toute une pensée, au niveau du style et de la forme, elle est en soi, déjà, expression, éclosion verbale, si j'ose dire, d'une vision du monde. L'écriture est ici le premier laboratoire dans lequel se craquelle un vernis de mauvais aloi, celui du monde réel.

Il va de soi que tous ces effets ne pouvaient être nivelés ou rabotés dans la version française sous peine de fausser toute une pensée, toute une philosophie et une éthique.

Or, il est à mon sens un facteur incontournable pour le traducteur qui veut échapper à la menace de la «normalisation»: l'empathie. Il se produit souvent chez le «bon» traducteur un phénomène indéniable de mimétisme et d'identification avec le texte dont il capte «la voix». L'empathie n'a rien à voir avec une quelconque parenté idéologique, et l'on peut d'ailleurs apprécier un texte tout en n'aimant pas son auteur. Je me réfère plutôt à une ressemblance dans l'approche des choses, à une analogie de tempérament qui conduira à une même sensibilité générale. Bref, à une «même longueur d'ondes», ondes qui viennent modeler le style. Et comme Valéry Larbaud, j'aime dire: «Dis-moi qui tu traduis, et je te dirai qui tu es...». L'empathie que j'ai ressentie pour Ernst Bloch ou pour Jean Améry n'avait rien à voir avec le juif marxiste 
d'un côté, la victime existentialiste de l'holocauste de l'autre. L'identification se situerait plutôt sur le plan des affects, affects transcendés ou esthétiquement sublimés qui se décantent dans la mélodie et le rythme textuels. C'est ainsi que je n'ai eu aucune difficulté à rendre le souffle enthousiaste et l'énergie vitale de Bloch que j'ai toujours ressentis à l'œuvre dans ma propre expression orale ou écrite. Que d'autre part je me suis toujours senti des affinités profondes avec ce qui sous-tend tous les textes d'Améry: à savoir une logique implacable et la sarcastique amertume suscitée par le mal. Voilà donc des registres qu'il m'est facile d'entonner. C'est pour toutes ces raisons sans doute qu'un pianiste jouant Chopin avec brio ne sera pas nécessairement aussi génial dans une interprétation de Bach.

En conclusion: le traducteur littéraire ou poétique échappera à la menace de la normalisation s'il cesse de se complaire dans le carcan de sa langue, et s'il la traite au contraire comme un organe vivant, porteur de germes enfouis mais susceptibles d'être développés, un organe souple capable de se laisser transformer sans se laisser casser ou détériorer; s'il la considère comme une terre d'accueil désireuse de récolter d'autres visions du monde et d'autres esthétiques; si, conscient de tout cela, il est lui-même assez talentueux ou doué pour créer une seconde fois - avec tout ce que cela implique d'audace et de risques - ce que son auteur a su créer de toutes pièces, et s'il comprend que sa fidélité doit être double, et concernera non seulement la culture et la langue réceptrices, mais aussi, et même surtout, celles qu'il a pour vocation de transposer. La langue qu'il créera alors sera une langue troisième, un "entre-deux», qui laissera transparaître l'étranger, l'Autre, mais sans défigurer le matériau d'accueil, dosage subtil et délicat qu'il lui faudra apprendre à maîtriser. Et le jeu en vaut bien la chandelle, puisque cette attitude profondément altruiste et tolérante, consistant à transformer son propre terrain linguistique en terre d'accueil véritable, fera de ce traducteur de bon aloi le plus authentique des ambassadeurs culturels. 